\title{
A survey of proteomic biomarkers for heterotopic ossification in blood serum
}

\author{
Laura E. Edsberg $^{1 *}$, Erin L. Crowgey ${ }^{2}$, Patrick M. Osborn ${ }^{3}$ and Jennifer T. Wyffels ${ }^{4}$
}

\begin{abstract}
Background: Heterotopic ossification $(\mathrm{HO})$ is a significant problem for wounded warriors surviving high-energy blast injuries; however, currently, there is no biomarker panel capable of globally characterizing, diagnosing, and monitoring $\mathrm{HO}$ progression. The aim of this study was to identify biomarkers for $\mathrm{HO}$ using proteomic techniques and blood serum.

Methods: Isobaric tags for relative and absolute quantitation (iTRAQ) was used to generate a semi-quantitative global proteomics survey of serum from patients with and without heterotopic ossification. Leveraging the iTRAQ data, a targeted selection reaction monitoring mass spectrometry (SRM-MS) assay was developed for 10 protein candidates: alkaline phosphatase, osteocalcin, alpha-2 type I collagen, collagen alpha-1 $(\mathrm{V})$ chain isoform 2 preprotein, bone sialoprotein 2, phosphatidate phosphatase LPIN2, osteomodulin, protein phosphatase 1J, and RRP12-like protein.

Results: The proteomic survey of serum from both healthy and disease patients includes 1220 proteins and was enriched for proteins involved in the response to elevated platelet $\mathrm{Ca}^{+2}$, wound healing, and extracellular matrix organization. Proteolytic peptides from three of the ten SRM-MS proteins, osteocalcin preprotein, osteomodulin precursor, and collagen alpha-1 (v) chain isoform 2 preprotein from serum, are potential clinical biomarkers for HO.

Conclusions: This study is the first reported SRM-MS analysis of serum from individuals with and without heterotopic ossification, and differences in the serum proteomic profile between healthy and diseased subjects were identified. Furthermore, our results indicate that normal wound healing signals can impact the ability to identify biomarkers, and a multi-protein panel assay, including osteocalcin preproprotein, osteomodulin precursor, and collagen alpha-1(v) chain isoform 2 preprotein, may provide a solution for $\mathrm{HO}$ detection and monitoring.
\end{abstract}

Keywords: Heterotopic ossification, SRM-MS, Osteocalcin, Osteomodulin, Collagen alpha-1(v)

\section{Background}

Heterotopic ossification $(\mathrm{HO})$ is the formation of mature lamellar bone in nonosseous (soft) tissues [1]. HO has been associated with war injuries since World War I and is now recognized as a significant comorbidity for wounded warriors surviving high-energy blast injuries [1-3]. A study of combat-related extremity injuries in Operation Enduring Freedom (OEF) and Operation Iraqi Freedom (OIF) found the risk of $\mathrm{HO}$ is highest following a blast mechanism injury and an amputation within the zone of injury [1]. HO in the military population often results in chronic pain, difficulties fitting prostheses, joint ankylosis, functional limitations, prolonged rehabilitation, and substantial

\footnotetext{
* Correspondence: Ledsberg@daemen.edu

${ }^{1}$ Center for Wound Healing Research, Natural Sciences, Daemen College,

Amherst, NY 14226, USA

Full list of author information is available at the end of the article
}

morbidity $[4,5]$. Rates of $\mathrm{HO}$ in combat-related extremity injuries are greater than $60 \%[1,2]$.

In civilian populations, $\mathrm{HO}$ may occur after a traumatic event, including hip arthroplasty, distal humerus fractures, spinal cord injuries (SCI), and closed brain injuries [6]. The etiology of $\mathrm{HO}$ remains unknown, but clinical risk factors include trauma, amputation, traumatic brain injury (TBI), SCI, thermal injury, major hip arthroplasty, and other major orthopedic surgery [7]. The exact cellular events leading to $\mathrm{HO}$ are not yet identified and as a result, treatment has been limited to nonsteroidal anti-inflammatory (NSAID) drugs and local radiation therapy used prophylactically. Many patients require one or more surgical excisions of ectopic bone [7]. Individuals with combat injuries often have additional diagnoses for which these prophylactic treatments 
are contraindicated further limiting treatment options. NSAIDs can cause severe gastrointestinal problems, renal toxicity, and platelet deficiency. Similarly, radiation therapy carries significant risks including fracture nonunion, genetic mutation, malignancy, and reproductive organ damage. No current pharmaceutical treatment is approved by the FDA to treat $\mathrm{HO}$ [7].

The etiology of $\mathrm{HO}$ is not well characterized. Aberrant bone growth associated with the disease is diagnosed using radiographs. In order to prevent the development of $\mathrm{HO}$, early detection of biomarkers associated misregulated wound healing mechanisms is necessary. Serum is readily available and easily accessible for repeated sampling for biomarker identification, and robust serum biomarkers have been established for other disorders, including cardiovascular disease [8]. The development of $\mathrm{HO}$ requires a cell capable of bone production, an osteoinducive factor, and an environment supportive of osteoinduction [7]. Researchers have evaluated changes in proteins associated with osteoinduction in the serum following traumatic brain injury (TBI) in rats and humans [9], and serum from TBI patients accelerated the proliferation of osteoblastic differentiation in cells from human muscle [10]. In contrast to blood serum, tissue biopsy relative to the lesion samples are invasive and location of the biopsy can impact results. Collectively, this work supports the use of serum for the identification of markers associated with the development of HO.

Recent advancements in mass spectrometry (MS) technology have enabled proteomic analysis of complex biological samples and have aided in the identification of potential biomarkers in various diseases, including evaluation of bone metabolism [11]. A high-throughput MS technique, isobaric tags for relative and absolute quantitation (iTRAQ), enables a global analysis of the proteome differences between biological samples, which provides the foundation for identifying potential biomarkers, but additional quantitative assays are required. Proteomic differences identified using iTRAQ are expressed as a ratio between samples and are therefore relative and semi-quantitative. There are several quantitative assays available for protein biomarker analysis, such as antibody approaches used in ELISAs or peptide approaches used in advanced mass spectrometry assays. Antibody-based approaches are limited based on reagent specificity and availability, whereas a targeted MS assay is limited only by the proteolytic and ionization characteristics of the protein of interest. One type of MS assay, selection reaction monitoring (SRM-MS), is an advanced proteomics technology enabling the identification and precise quantification of peptides with high sensitivity, specificity, and reproducibility [12, 13]. Analytical information from peptides obtained using SRM-MS allows by inference, quantification of the corresponding proteins in complex biological samples. This technique is capable of producing high-quality diagnostic data in disease processes [14] and is a more sensitive and reproducible method for quantifying low-abundance proteins in complex biological samples. SRM-MS uses synthetic peptides to optimize detection transition parameters for each peptide target and as such is not an appropriate method for a high-throughput proteomics analysis. The objectives of this study were to develop an SRM-MS assay specific for overexpressed proteins present in the serum of subjects with $\mathrm{HO}$ and test their predictive ability using serum from subjects with and without $\mathrm{HO}$.

\section{Methods}

\section{Subject enrollment}

This study was part of a larger project to study the proteomics of $\mathrm{HO}$ in tissue and serum (manuscript in preparation), focusing on mass spectrometry analyses of serum samples from 41 subjects. Subjects were eligible for enrollment in the study if they had or were being treated for high-risk fractures, acetabular fracture, burns with orthopedic injury, traumatic brain injury with extremity trauma, undergoing amputation, and excision of ectopic bone or major arthroplasty. Subjects below the age of 18 or currently being treated for cancers or metastatic disease involving the bone were excluded.

\section{Study protocol and overview}

Participants were enrolled prior to surgery, and outcome was determined by evaluation of $x$-rays collected at the time of surgery and during follow-up visits at 6 weeks, 12 weeks, 6 months, and 12 months. Blood $\left(5 \mathrm{~cm}^{3}\right)$ was collected at time of the patient's scheduled surgical procedure. Blood samples were collected into sterile vacutainer tubes with clot activator and gel for serum separation. Serum was removed after $30 \mathrm{~min}$ to a sterile screw top polypropylene tube and snap frozen in liquid nitrogen before storage at $-80{ }^{\circ} \mathrm{C}$. Samples were assigned to $\mathrm{HO}$-positive or $\mathrm{HO}$-negative group-based disease status at the time of surgery and blood collection. Blood serum samples were pooled in equal volumes by disease status and analyzed using iTRAQ. Individual serum samples were analyzed for the abundance of specific proteins identified in the iTRAQ results using SRM-MS, a targeted quantitative analysis (Fig. 1).

\section{Sample and peptide preparation for ITRAQ}

Reagents were purchased from Sigma-Aldrich (St. Louis, MO) unless otherwise indicated. The top 14 most abundant proteins in the serum were depleted using the Seppro IgY14 column systems: albumin, IgG, $\alpha 2$-antitrypsin, IgA, IgM, transferrin, haptoglobin, $\alpha 2$-macroglobulin, fibrinogen, complement C3, $\alpha 1$-acid glycoprotein (orosomucoid), 


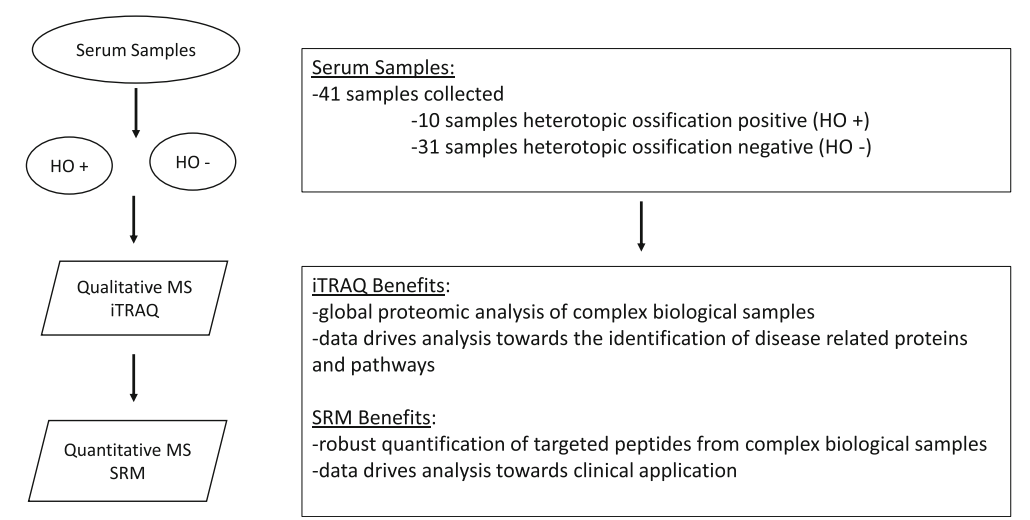

Fig. 1 Sample processing overview. A total of 41 serum samples were collected at time of surgery from subjects with $(n=10)$ and without $(n=31)$ heterotopic ossification. Serum samples were pooled by disease state and subjected to an isobaric tag for relative and absolute quantitation (iTRAQ) mass spectrometry (MS) analysis. The iTRAQ data was used to drive the selection of specific proteins to target via a selected reaction monitoring (SRM) MS technique, a qualitative MS technique that enables the robust quantification of specific peptides within a single subject's serum sample

HDL (apolipoproteins A-I and A-II), and LDL (mainly apolipoprotein B). Serum samples were diluted $5 \times$ in IgY dilution buffer, filtered $(0.22 \mu \mathrm{m})$, and then injected into IgY LC10 columns attached to an Agilent 1200 HPLC system. The unretained fraction was collected.

In-solution depleted serum samples were further processed by MyOmicsDx, Inc (Towson, MD, USA) using "filter-assisted sample preparation" (FASP) method [15]. Briefly, protein samples in $9 \mathrm{M}$ urea were reduced with $5 \mathrm{mM}$ tris-(2-carboxyethyl) phosphine (TCEP) at $37^{\circ} \mathrm{C}$ for $45 \mathrm{~min}$ and reduced cysteines were blocked using $50 \mathrm{mM}$ IAA at $25{ }^{\circ} \mathrm{C}$ for $15 \mathrm{~min}$. Protein samples were cleaned using $10 \mathrm{kDa}$ Amicon Filter (UFC501096, Millipore) three times using $9 \mathrm{M}$ urea and two times using MyProt-Buffer 1 (MyOmicsDx, Inc). Samples were proteolyzed with trypsin (V5111, Promega) for $12 \mathrm{~h}$ at $37^{\circ} \mathrm{C}$.

The peptide solution was acidified by adding $1 \%$ trifluoroacetic acid (TFA) and incubated at room temperature for $15 \mathrm{~min}$. A Sep-Pak light C18 cartridge (Waters Corporation) was activated by loading $5 \mathrm{~mL}$ $100 \%(\mathrm{vol} / \mathrm{vol})$ acetonitrile and washed by $3.5 \mathrm{~mL} 0.1 \%$ TFA solution two times. The acidified digested peptide solution was centrifuged at $1800 \times g$ for $5 \mathrm{~min}$, and the supernatant loaded into the cartridge. To desalt the peptides bound to the cartridge, 1,3 , and $4 \mathrm{~mL}$ of $0.1 \%$ TFA were used sequentially. To elute the peptides from the cartridge, $2 \mathrm{~mL}$ of $40 \%$ ( $\mathrm{vol} / \mathrm{vol})$ acetonitrile with $0.1 \%$ TFA was used. The eluted peptides were lyophilized overnight and reconstituted in $37 \mu \mathrm{L}$ MyProtBuffer 3 (MyOmicsDx, Inc, Towson, MD, USA).

\section{Multiplexed iTRAQ labeling}

Digested peptides from samples in a volume of $37 \mu \mathrm{l}$ MyProt-Buffer 2 were labeled using 4-plex iTRAQ reagents (ABSciex, Framingham, MA, USA). After 2 h, labeled peptides were dried to remove organic solvents and reconstituted in $500 \mu \mathrm{l}$ MyProt-Buffer 3 (MyOmicsDx, Inc, Towson, MD, USA), combined and fractionated on a bRPLC (basic reverse phase liquid chromatography) column (XBridge BEH C18 Column, $5 \mu \mathrm{m}, 2.1 \times 100 \mathrm{~mm}$ ) via XBridge BEH C18 Guard Column (Waters Corporation) using an Agilent 1260 HPLC system. Peptides in each fraction were dried and re-suspended in $8 \mu \mathrm{l} 0.1 \%$ formic acid (EMD Millipore, Billerica, MA, USA) with 3\% acetonitrile for LC-MS/MS analysis.

A Sep-Pak light C18 cartridge (Waters Corporation) was activated by loading $5 \mathrm{~mL} \mathrm{100 \%} \mathrm{(vol/vol)} \mathrm{acetonitrile}$ (JT Baker) and was washed by $3.5 \mathrm{~mL} \mathrm{0.1 \%} \mathrm{TFA} \mathrm{solu-}$ tion two times. Acidified digested peptide solution was centrifuged at $1800 \mathrm{~g}$ for $5 \mathrm{~min}$, and the supernatant was loaded into the cartridge. To desalt the peptides bound to the cartridge, 1,3 , and $4 \mathrm{~mL}$ of $0.1 \%$ TFA were used sequentially. To elute the peptides from the cartridge, $2 \mathrm{~mL}$ of $40 \%$ ( $\mathrm{vol} / \mathrm{vol}$ ) acetonitrile with $0.1 \%$ TFA was used, and this elution was repeated two more times for a total of $6 \mathrm{~mL}$ of eluate. It was important to ensure that the cartridge had stopped dripping before each sequential wash and elution solution was applied. The eluted peptides were lyophilized overnight and reconstituted in $37 \mu \mathrm{L}$ of MyProt-Buffer 2 (MyOmicsDx, Inc, Towson, MD, USA).

\section{Nanoflow electrospray ionization tandem mass spectrometry analysis}

Data-dependent MS/MS analyses of the iTRAQ-labeled peptides were carried out by MyOmicsDx, Inc. (Towson, MD) on a $\mathrm{Q}$ Exactive $^{\mathrm{Tx}}$ Hybrid Quadrupole-Orbitrap Mass Spectrometer (https://www.thermofisher.com/us/ en/home.html) interfaced with Proxion nanoflow LC system. Peptides were fractionated by reverse phase HPLC on a $75 \mu \mathrm{m} \times 15 \mathrm{~cm}$ PicoFrit column packed with Magic C18AQ (5 $\mu \mathrm{m}, 120 \AA$, https://www.bruker.com/) 
using $0-60 \%$ acetonitrile $/ 0.1 \%$ formic acid gradient over $90 \mathrm{~min}$ at $300 \mathrm{~nL} / \mathrm{min}$. Eluting peptides were sprayed directly into Q Exactive $\mathrm{e}^{\mathrm{Tw}}$ at $2.0 \mathrm{kV}$.

Survey scans (full MS) were acquired from 350 to $1800 \mathrm{~m} / \mathrm{z}$ with up to 15 peptide masses (precursor ions) individually isolated with a 2-Da isolation window and fragmented (MS/MS) using a collision energy of $29 \%$ and $30 \mathrm{~s}$ dynamic exclusion. Precursor and the fragment ions were analyzed at 70,000 and 17,500 resolutions, respectively. Peptide sequences were identified from isotopically resolved masses in MS and MS/MS spectra extracted with and without de-convolution using Thermo Scientific MS2 processor and Xtract software.

\section{ITRAQ-MS data processing}

Mass spectrometry raw files were automatically processed through Proteome Discoverer 2.1 software using Xtract and MS2-processor spectrum processor in addition to default spectrum selector node. The data was searched in Refseq 2015 human entries using Mascot search engine interfaced with different processing nodes of Proteome Discoverer 2.1. Search parameters included oxidation on methionine, iTRAQ 4-plex on tyrosine, deamidation on residues $\mathrm{N}$ and $\mathrm{Q}$ as different variable modifications, iTRAQ 4-plex on N-terminus and lysine residue, and methylthio on cysteine residue as different fixed modifications. Mass tolerances on precursor and fragment masses were set to $15 \mathrm{ppm}$ and $0.03 \mathrm{Da}$, respectively. Peptide validator node was used for peptide validation with stringent cutoff of 0.01 and relaxed cutoff of 0.05 false discovery rate (FDR), and 1\% FDR cutoff was used to filter the data.

High confidence ( $0.1 \%$ FDR) and top ranked peptides were considered with protein grouping options. Protein ratios were normalized through MyProt-QuantiR (MyOmicDx, Inc) software package, and peptides with $>30 \%$ isolation interference were excluded from protein quantification to avoid potential interference of reporter ions from contaminant peaks. MA plots were used to evaluate any potential bias between quantification channels within experiment and between experiments.

\section{Proteomic bioinformatics analysis}

The entire iTRAQ dataset, regardless of protein ratio, was uploaded into Cytoscape v3.3.0 [16] and analyzed using the ReactomeFI plugin (database 2015) [17] to generate an interactome, followed by a pathway enrichment analysis. Gene ontology enrichment analysis for biological process, cellular component, and molecular function were completed using the cytoscape app BiNGO [18] and REVIGO [19]. The iTRAQ ratio (expression) data was overlaid with biological annotations to prioritize proteins for SRM analysis.

\section{Reagents SRM-MS}

TCEP (tris-(2-carboxyethyl) phosphine) was purchased from Thermo Scientific (Waltham, MA). LysC and trypsin proteases were purchased from Promega (Fitchburg, WI). C18 Cartridges for sample preparation and chromatography columns for bRPLC and online HPLC of Triple Quadrupole mass spectrometer were purchased from Waters (Milford, MA). Acetonitrile was purchased from JT Baker, and formic acid was obtained from EMD Millipore (Billerica, MA, USA). MyProt-Buffer 1, MyProt-Buffer 2, and MyProt-Buffer 3 were utilized by MyOmicsDx, Inc (Towson, MD, USA). All other reagents were purchased from Sigma-Aldrich (St. Louis, MO) unless otherwise indicated.

\section{SRM-MS data processing}

Peptide samples reconstituted in 37ul MyProt-Buffer 3 (MyOmicsDx, Inc) were spiked with MyProt-SRM Internal Control Mixture (MyOmicsDx, Inc) composed of a pool of $1 \mathrm{f}$ mole heavy isotope-labeled peptides covering a large hydrophobicity window and a large $\mathrm{M} / \mathrm{z}$ range $(\mathrm{M} / \mathrm{z} 200 \sim 1300)$ and were subject to SRM analysis. Peptide samples were eluted through an online Agilent 1290 HPLC system into the Jet Stream ESI source of an Agilent 6495 Triple Quadrupole Mass spectrometer.

Thirty peptides representing 10 proteins were chosen as SRM targets from MyOmicsDx's manually curated SRM target peptide database, MyProt-SRM Map, based on their ITRAQ ratio. Transition parameters and retention times of the 30 peptides were confirmed individually using an Agilent 6495 Triple Quadrapole Mass Spectrometer for both doubly and triply charged precursor ions. Five or 6 transitions per peptide precursor were selected for SRM analysis.

Three hundred and fifty sets of transition parameters (corresponding to 30 peptides, representing the abundance of 10 proteins) and 30 SRM data files containing the quantitative data of 30 peptides in 30 human serum samples (Additional file 1) were imported into Skyline 3.1 [20]. The abundance of each target peptide was represented by the total area under the curve (AUC) of all its transitions normalized to the total AUC of all transitions from the most nearby (sharing a similar hydrophobicity) heavy isotope-labeled peptide from MyProt-SRM Internal Control Mixture (MyOmicsDx, Inc) spiked in before the SRM analysis. The abundance of each target peptide was represented by the total AUC of all its transitions normalized to the total AUC of each control peptide's transitions. The relative abundance level of a target peptide in different samples was represented by its relative ratio to the abundance level of the internal control peptide in the same sample. The abundance dataset was further normalized by the ratio 
obtained from a subject without HO, (ND-1), chosen randomly as a reference disease negative sample in this study. Differences in SRM peptide abundance were tested using $\mathrm{R}$ and Welch's $t$ test.

\section{Model construction}

The dataset obtained from the SRM-MS assay was composed of 30 samples, $10 \mathrm{HO}$ positive and $20 \mathrm{HO}$ negative, with 17 peptide abundance values for each sample. The number of samples in this pilot study was limited, and the number of parameters $(p)$ for each subject $(n)$ is relatively large compared to the total number of samples $(n=30)$. The SRM assay dataset was analyzed using three models, random forest (RF), generalized linear model (GLM), and support vector machine learning (SVM).

Random forest, originally proposed by Breiman in 1999 , is an ensemble classification algorithm composed of a series of decision trees. Each tree is built independently through a technique called "bagging" based on random selection of input variables. The prediction result is based on the vote made by all trees. This modeling approach provides a very accurate classifier, but it has not been widely used in clinical diagnostics. In contrast, logistic regression is a widely used standard regression model for binary data. It has been widely used to construct biomarker panels for clinical diagnostics. Support vector machine learning is a supervised machine learning model that identifies the optimal separating hyperplane between two classes or states based on least-squares regression of the data.

Seventy percent of the dataset was used to construct models, and thirty percent of the dataset was withheld to evaluate model performance. Resampling, regression, and prediction were repeated 1000 times for each model. Model performance was evaluated by comparing the results generated from at least 100 predictions for each of the three models, and the subset of models used to do the prediction in each category were preselected with a AUC of ROC no smaller than 0.98 .

\section{Results}

\section{iTRAQ results}

\section{Proteomics analysis and serum biomarker selection}

In total, 41 subjects including 27 men and 14 women ranging in age from 22 to 83 were enrolled (Table 1). HO-negative samples were derived largely from total hip arthroplasty (ages 28-83) in both male and female subjects ages 22-83. All HO-positive samples were collected during $\mathrm{HO}$ excision or hip revision procedures in men and women ages $22-40$. Serum samples were analyzed via qualitative proteomics analysis and/or a targeted quantitative analysis (Fig. 1). The high-throughput proteomics analysis consisted of a 4-plex iTRAQ experiment, which utilized separately pooled serum samples from
HO-positive $(n=10)$ and HO-negative $(n=31)$ subjects. No bias between biological or technical replicates was observed, and median normalization was applied to the raw data to allow direct comparison of the reported ratios of the proteins between $\mathrm{HO}$-positive and $\mathrm{HO}$ negative groups. Collectively, 1220 unique proteins (UniProtKB Accession) were measured, and a ratio per protein was calculated between separately pooled $\mathrm{HO}$ positive and $\mathrm{HO}$-negative serum samples. The majority of the proteins had an expression ratio between 0.5 and 1.5 (data not shown).

The interactome (Fig. 2) and subsequent pathway enrichment analysis (Table 2) indicated extracellular matrix organization, ECM-receptor interaction, response to elevated platelet cytosolic $\mathrm{Ca}^{+2}$, and complement and coagulation cascades were enriched. The gene ontology biological process enrichment analysis included response to wounding, acute inflammatory response, and activation of plasma proteins involved in acute inflammatory response as top enriched biological processes (Fig. 3; and Additional file 2).

In total, 10 candidate proteins were selected for SRMMS from the ITRAQ studies based on relative fold changes in ITRAQ ratios and the characteristics of the proteotypic peptides for each protein (Table 3). Eight proteins, osteomodulin (OMD), collagen alpha-2(l) chain precursor (COL1A2), collagen alpha-1(V) chain isoform 2 preprotein (COL5A1), alkaline phosphatase (ALPL), phosphatidate phosphatase LPIN2 (LPIN2), RRP12-like protein (RRP12), TRAF3-interacting protein 1 (TRAF3), and protein phosphatase 1J (PPM1J), were selected from the serum iTRAQ results and subsequent bioinformatics analysis. Two additional proteins, bone sialoprotein 2 precursor (IBSP) and osteocalcin preprotein (BGLAP), were selected from an iTRAQ survey of tissue samples from $\mathrm{HO}$-positive and HO-negative samples from the same study subjects because the ratio reported from the tissue data suggested a significant increase of this protein in the disease state compared to non-disease (manuscript in preparation).

\section{SRM analysis}

SRM transition parameters of all 30 peptides targeting the top overexpressed proteins quantified by iTRAQ were incorporated into a SRM-MS assay method. Each protein was independently quantified by 3 peptides in 30 individual patient serum samples. Seventeen peptides representing 9 of the 10 proteins from the initial experimental design, alkaline phosphatase, osteocalcin, alpha-2 type I collagen, collagen alpha- $1(\mathrm{~V})$ chain isoform 2 preprotein, bone sialoprotein 2, osteomodulin, protein phosphatase IJ, and RRp12-like protein, were validated as SRM targets after successfully being detected and quantified in serum (Additional file 3). Phosphatidate 
Table 1 Surgical procedures and heterotopic ossification status of serum samples

\begin{tabular}{|c|c|c|c|c|c|c|c|c|}
\hline & \multicolumn{4}{|c|}{$\mathrm{HO}$ negative } & \multicolumn{4}{|c|}{ HO positive } \\
\hline & Subjects & M & $\mathrm{F}$ & Age & Subjects & M & $\mathrm{F}$ & Age \\
\hline All & 31 & 18 & 13 & $22-83$ & 10 & 9 & 1 & $22-40$ \\
\hline Total hip arthroplasty (THA) & 18 & 10 & 8 & $28-83$ & - & & & \\
\hline Open reduction and internal fixation (ORIF) & 7 & 5 & 2 & $26-64$ & - & & & \\
\hline Hip revision (HR) & 3 & & 3 & $45-62$ & 2 & 1 & 1 & $36-40$ \\
\hline HO excision (HOE) & - & & & & 8 & 8 & & $22-31$ \\
\hline Other & 3 & 3 & & $22-36$ & - & & & \\
\hline
\end{tabular}

A total of 31 serum samples were collected from subjects (18 male and 13 female) with wounds but no signs of disease, heterotopic ossification negative (HO-). A total of 10 serum samples were collected from heterotopic ossification positive (HO+) subjects (9 male and 1 female). Serum samples were collected at time of surgery. The surgical procedure (or wound) differed between subjects, with the most common procedures being total hip arthroplasty (THA), open reduction and internal fixation, hip revision, and $\mathrm{HO}$ excision. Subjects ranged in age from 22 to 83 years old

phosphatase LPIN2 isoform X2 was detectable only by one peptide in five $\mathrm{HO}$ - and five $\mathrm{HO}+$ samples and was dropped from the panel.

The RF, GLM, and SVM algorithms produced predictive models that were comparable in performance (Fig. 4), but RF predictions were closest to the true disease state for 9 of the $10 \mathrm{HO}+$ subjects (binary value where 0 indicates $\mathrm{HO}$ negative or non-disease (ND) and 1 indicates $\mathrm{HO}$ positive or disease (D)) (Fig. 4). Using the RF generated model, the SRM peptides were ranked by the mean square error (MSE) increase if the peptide is randomly permuted. If a peptide is an important predictor, then the model fit decreases when it is randomly permuted and the overall MSE increases. Peptides SRM-8, SRM13, SRM-4, SRM-5, and SRM-6 representing proteins osteocalcin preprotein, osteomodulin precursor, and collagen alpha-1(v) chain isoform 2 preprotein were identified as potential biomarkers for $\mathrm{HO}$ (Fig. 5).

Relative expression levels of the peptide biomarkers were significantly different for SRM peptides, SRM-4, SRM-5, SRM-6, SRM-13, SRM-14, SRM-8, SRM-3, SRM-23, derived from osteocalcin, collagen alpha-1(V) chain, osteomodulin, bone sialoprotein 2, and RRP12like protein (Fig. 6). Three osteocalcin peptides (SRM4,

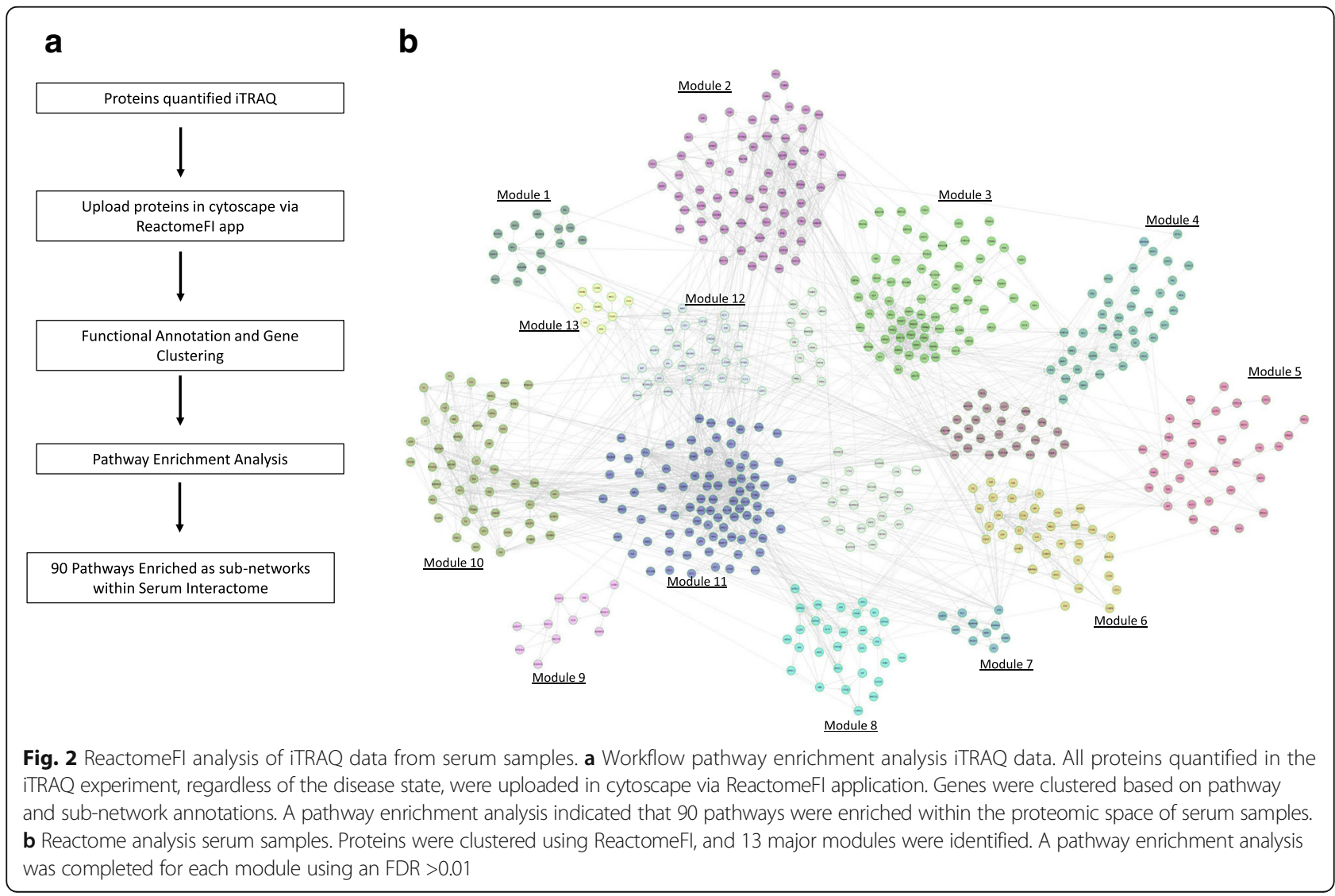


Table 2 Pathway enrichment summary iTRAQ data

\begin{tabular}{|c|c|c|c|c|c|c|}
\hline Biological pathway & Pathway database & Ratio & Pathway proteins & Dataset proteins & $p$ value & FDR \\
\hline Extracellular matrix organization & $R$ & 0.0243 & 248 & 64 & $1.11 \mathrm{E}-16$ & 1.30E-14 \\
\hline ECM-receptor interaction & K & 0.0085 & 87 & 31 & $1.11 \mathrm{E}-16$ & $1.30 \mathrm{E}-14$ \\
\hline Response to elevated platelet cytosolic $\mathrm{Ca}^{2+}$ & R & 0.0081 & 83 & 45 & $1.11 \mathrm{E}-16$ & 1.30E-14 \\
\hline Complement and coagulation cascades & K & 0.0068 & 69 & 46 & $1.11 \mathrm{E}-16$ & $1.30 \mathrm{E}-14$ \\
\hline Beta1 integrin cell surface interactions & N & 0.0065 & 66 & 27 & $1.11 \mathrm{E}-16$ & 1.30E-14 \\
\hline Formation of fibrin clot (clotting cascade) & $\mathrm{R}$ & 0.0038 & 39 & 24 & $1.11 \mathrm{E}-16$ & 1.30E-14 \\
\hline Focal adhesion & K & 0.0203 & 207 & 37 & $8.46 \mathrm{E}-12$ & $8.46 \mathrm{E}-10$ \\
\hline Staphylococcus aureus infection & K & 0.0054 & 55 & 18 & $2.67 \mathrm{E}-10$ & 2.35E-08 \\
\hline Beta3 integrin cell surface interactions & N & 0.0042 & 43 & 16 & $4.32 \mathrm{E}-10$ & 3.37E-08 \\
\hline L1CAM interactions & $\mathrm{R}$ & 0.0077 & 79 & 20 & $2.15 \mathrm{E}-09$ & $1.38 \mathrm{E}-07$ \\
\hline
\end{tabular}

All proteins quantified in the iTRAQ experiment, regardless of the disease state, were uploaded in cytoscape via ReactomeFI application. A pathway enrichment analysis was executed for the entire reactome

$R$ Reactome, $K$ KEGG, $N$ NCI PID

5, and 6) had similar patterns between the disease and non-disease state, supporting a higher abundance in $\mathrm{HO}+$ compared to HO-. Collagen alpha 1 (SRM13, 14, 15) had two peptides (SRM13 and 14) that support this protein in higher abundance in $\mathrm{HO}$, whereas SRM15 showed no difference between $\mathrm{HO}-$ and $\mathrm{HO}+$. Two peptides were measured for osteomodulin (SRM7 and 8), and both peptides were elevated in $\mathrm{HO}+$ compared to $\mathrm{HO}-$. Two peptides were measured for alkaline phosphatase (SRM17 and 18), and SRM18 had greater dispersion in the upper quartile in $\mathrm{HO}+$ compared to $\mathrm{HO}-$. A single peptide was measured for bone sialoprotein (SRM3), and the data support higher peptide abundance in $\mathrm{HO}+$ compared to $\mathrm{HO}-$. A single peptide was measured for RRP12 (SRM 23) and demonstrated greater dispersion in expression in $\mathrm{HO}+$ versus $\mathrm{HO}-$ samples. One peptide was quantified for TRAF3 (SRM27), which showed no difference between $\mathrm{HO}+$ and $\mathrm{HO}-$.
Protein-protein interactions between SRM candidates were identified by ReactomeFI (Fig. 7) such that their connections with each other (panel a) and within the context of differentially regulated proteins in iTRAQ experiment (panel b) could be analyzed. Of interest, six of the SRM candidates, BGLAP, COL1A2, COL5A1, IBSP, LPIN2, and OMD, were clustered together using linker regions and are involved in extracellular matrix organization and ECM-receptor interaction (panel a). When these candidates were examined in conjunction with other differentially regulated proteins, response to elevated platelet cystosolic $\mathrm{Ca}^{+2}$ sub-network was enriched (panel b). Collectively, these data suggest that there are several sub-networks, which are highly connected through protein-protein interactions that contain proteins that are differentially expressed in $\mathrm{HO}$.

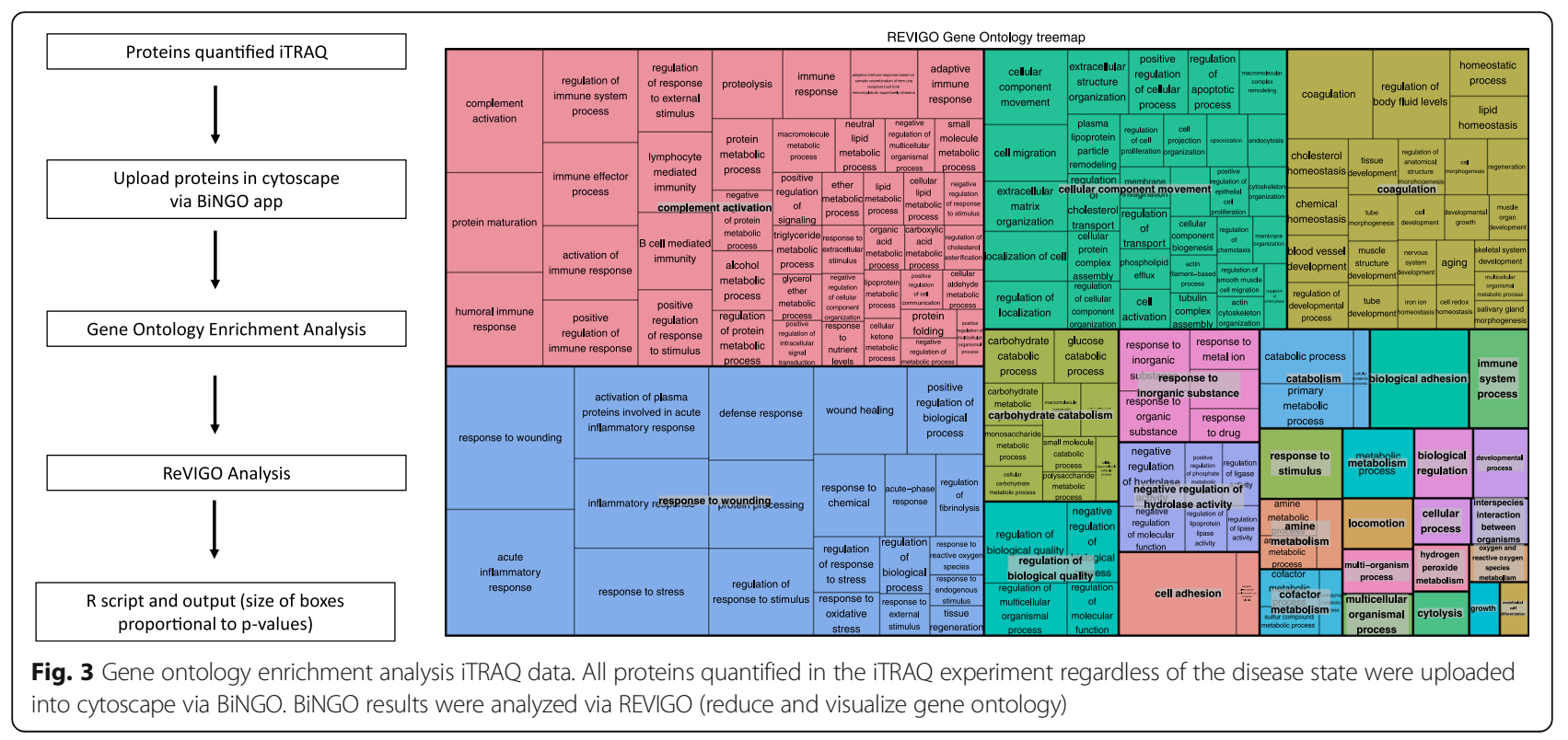




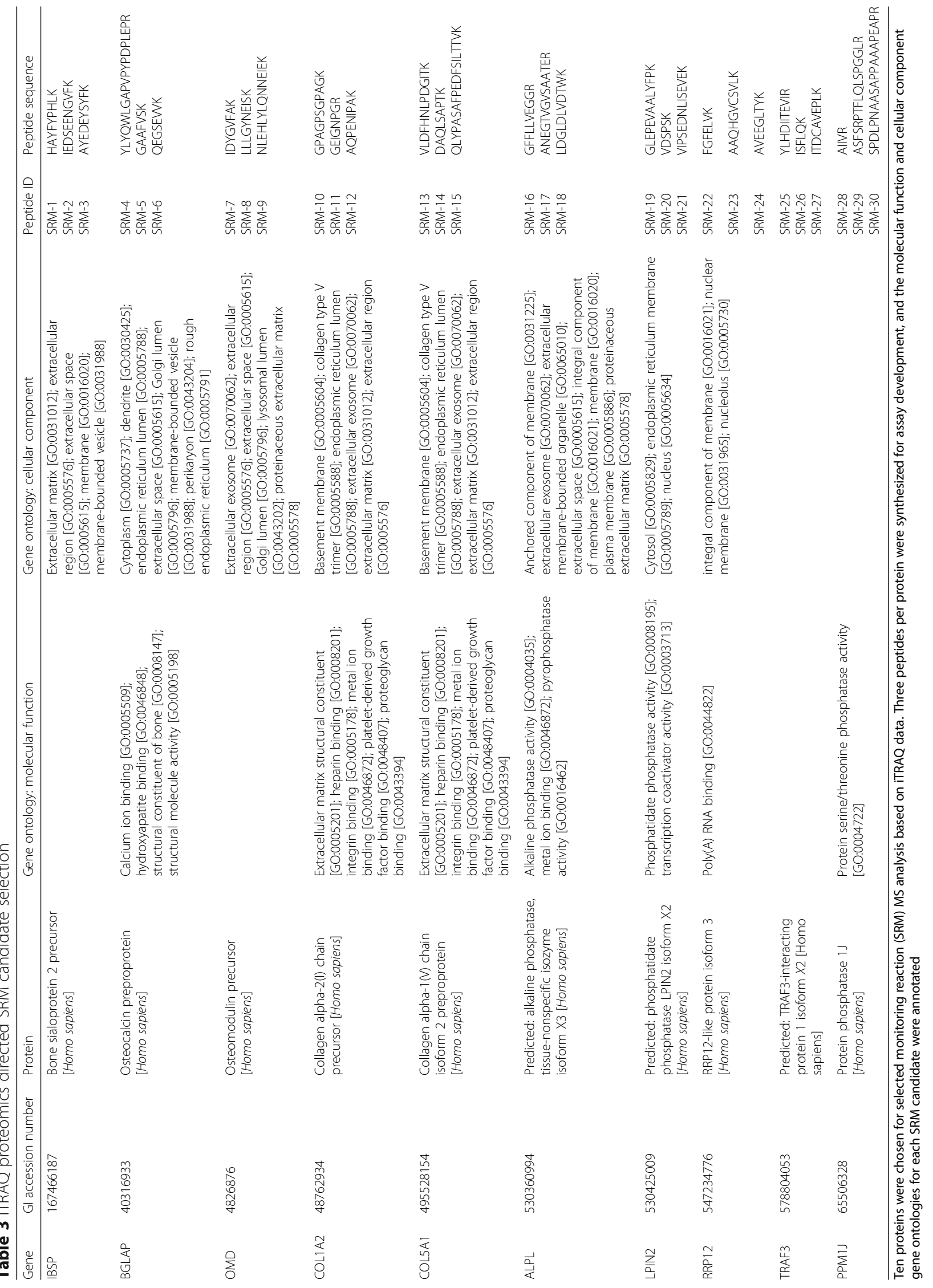




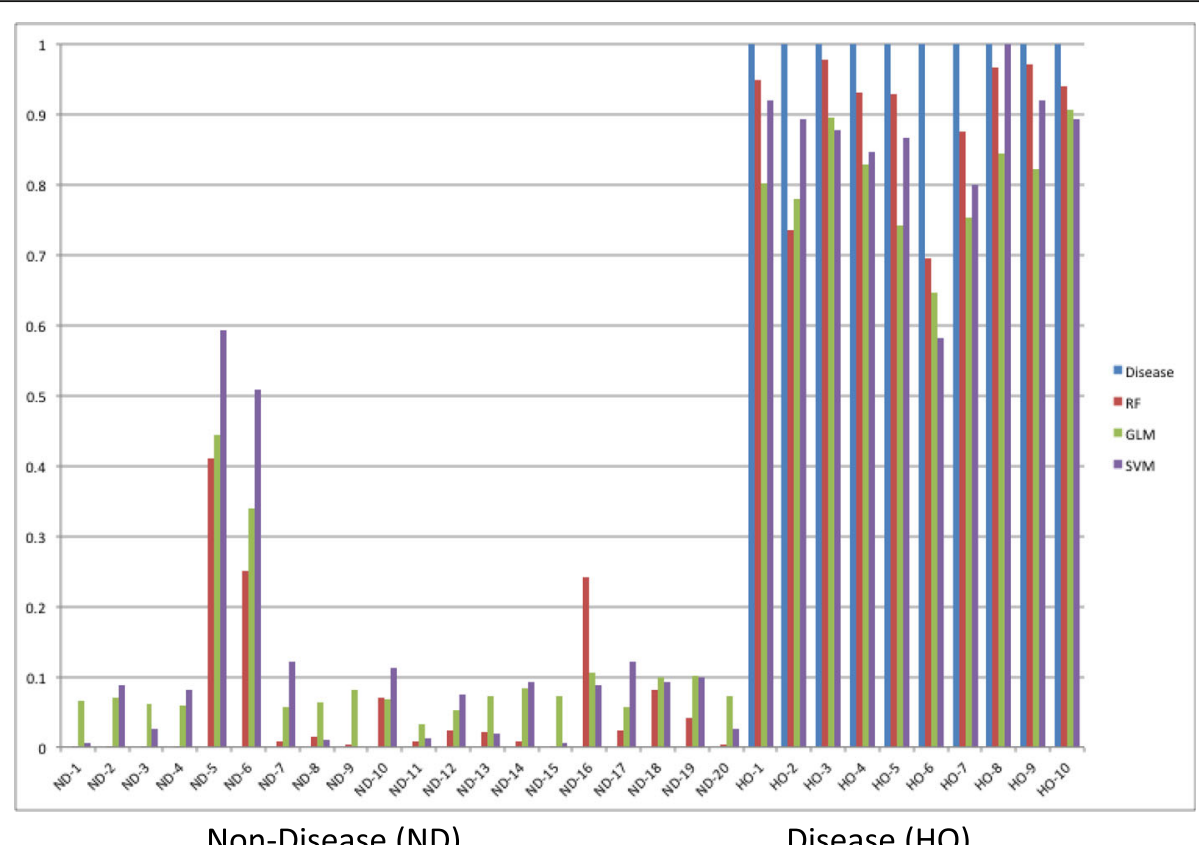

Fig. 4 Summary of model comparison SRM assay. Samples were annotated as either disease state (heterotopic ossification positive-HO+) or non-disease (ND) state (heterotopic ossification negative- - HO-). Three different statistical models were utilized to analyze the SRM-MS data: random forest (RF; red line), generalized linear model (GLM; green line), and support vector machine learning (SVM; purple line). A non-disease state for $\mathrm{HO}$ - prediction was 0 (blue line left panel) and a disease state for $\mathrm{HO}+$ prediction was 1 (blue line right panel). All three statistical models performed similarly

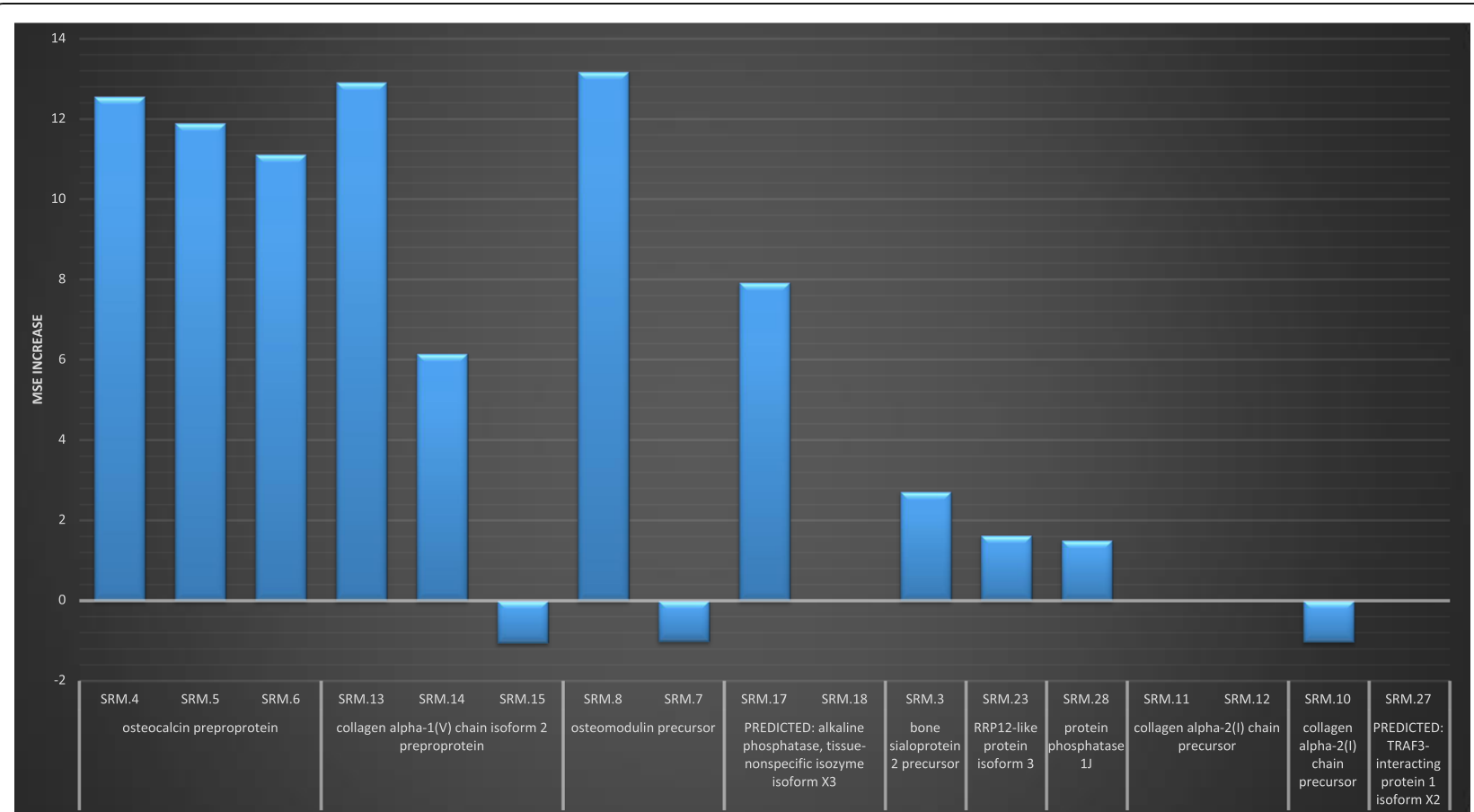

Fig. 5 Mean square error analysis for random forest model. Using the random forest (RF) model, peptides with a mean square error (MSE) increase $>8$ were considered important variables because random permutation of these variables had a significant impact on the model prediction of disease state versus non-disease state 


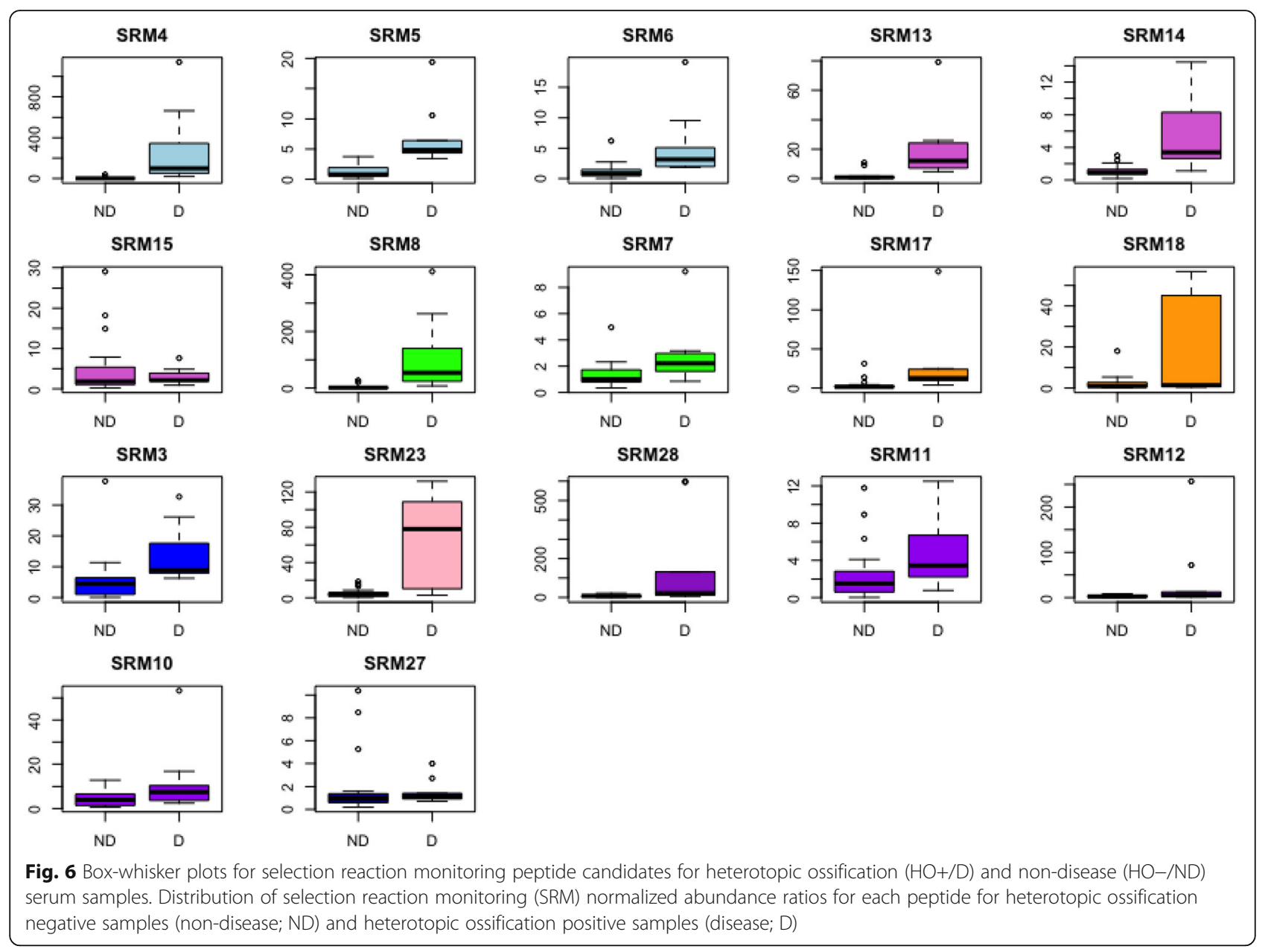

\section{Discussion}

The presence of $\mathrm{HO}$ is determined using radiographs, which limits the ability to predict patients that are susceptible to $\mathrm{HO}$ and complicates early diagnosis of the disease as aberrant bone formation must first be detectable. Furthermore, using tissue samples to identify early signs of $\mathrm{HO}$ can be difficult because the proteome of tissue within a wound bed can vary widely depending on location within the wound bed [21]. Using tissue for disease identification requires invasive sampling and like all biopsy results, is dependent on the location of the sample in relation to the suspected disease foci.

A biomolecular screening tool using serum from wounded patients could allow for earlier diagnosis, intervention, and the potential development of novel therapeutics, to prevent development of $\mathrm{HO}[2,9]$. A major challenge with identifying systemic markers is the need for data-driven approaches. Applying a priori knowledge limits the advancement of screening assays because the majority of protein candidates, for example, MMPs are involved in normal healing and disease processes [22]. Effective diagnostic panels require multiple biomarkers across different gene families because the disease state is more often a consequence of misregulation of protein expression rather than a single mutation of a critical protein.

Another challenge for devising a pharmaceutical treatment for $\mathrm{HO}$ is the lack of knowledge regarding metabolic processes and misregulated cellular signaling events underlying the disease. Since $\mathrm{HO}$ has similar characteristics as seen in the normal physiology of fracture healing, treatment options for $\mathrm{HO}$ need to be very specific to avoid impairment of normal bone healing [22]. Identifying biomarkers that allow for early identification of $\mathrm{HO}$ is confounded by the active and ongoing inflammatory response present due to injury. During the inflammatory phase of the wound healing process, without the formation of heterotopic ossification, there will be a strong signal in the biological space of proteins related to wound healing.

Utilizing a shotgun proteomics assay, iTRAQ, qualitative expression levels were determined for all detectable proteins (1220) from serum samples collected from $\mathrm{HO}$ + and $\mathrm{HO}-$ subjects and used to identify proteins that 


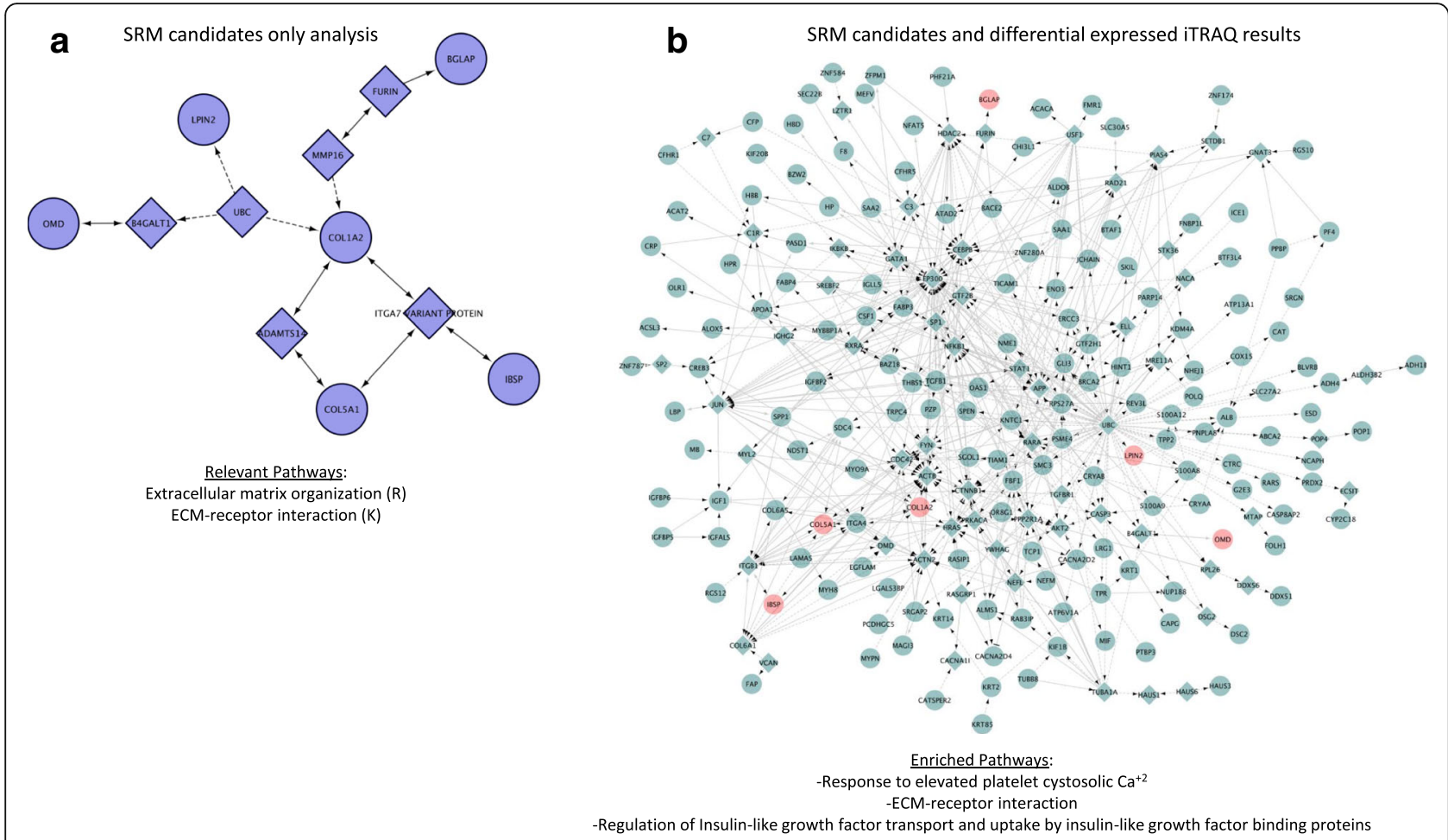

Fig. 7 Summary target candidates for selection reaction monitoring assay. a The 10 proteins used in the selection reaction monitoring (SRM) assay were analyzed via ReactomeFl in cytoscape. Six of the candidates (circles) were clustered with six linker genes (diamonds). Relevant pathways for wound healing and ossification within this small interactome were extracellular matrix organization and extracellular matrix-receptor interaction. b The 10 proteins (red nodes) used in the selection reaction monitoring (SRM) assay were analyzed via ReactomeFl in cytoscape with all proteins that were differentially regulated (green nodes) in the ITRAQ experiment

are differentially regulated between the disease and nondisease state. This global proteomics approach enabled a data-driven methodology. The bioinformatics analyses built networks of functionally related proteins capable of identifying crosstalk through protein-protein interactions between sub-networks. The goal of this approach was to identify biomarkers, proteins linked to misregulated pathways and that are differentially expressed in the disease state compared to non-disease state. The current research found that serum from both healthy and disease patients is enriched for proteins involved in the response to elevated platelet $\mathrm{Ca}^{+2}$, wound healing, and extracellular matrix organization, and that these pathways include proteins that are differentially regulated in the disease state (Table 2).

Shotgun proteomic techniques including iTRAQ provide a knowledgebase for identifying potential clinical biomarkers without the need for a priori knowledge, but results are semi-quantitative and require follow-up validation using a quantitative assay. To transition the semiquantitative iTRAQ results into a clinical diagnostic system, we developed and utilized SRM-MS assays to precisely and robustly quantify 10 proteins chosen based on expression ratios from the iTRAQ experiment combined with functional annotations, including gene ontology and pathway information. Using a random forest model and the SRM-MS data, osteocalcin preproprotein, osteomodulin precursor, and collagen alpha-1(V) chain isoform 2 preprotein were determined to be the best candidates for predicting the disease state $(\mathrm{HO}+)$.

The model predictions of these targets as diagnostic markers are supported by a study of osteoclast and osteoblast activity after total hip arthroplasty, which found that osteocalcin increased in individuals who developed HO [23]. Osteocalcin (gene BGLAP; P02818) is secreted by bone-forming osteoblasts [24], and a strong overexpression of osteocalcin mRNA in $\mathrm{HO}$ isolated cells has been observed [25]. The wound fluid from blast-injured patients has osteoinductive signaling properties [5]. Serum from patients with TBI induced an increase in skeletal muscle cells, and the high levels of alkaline phosphatases suggested an increased osteogenic capability [10].

Bone formation and remodeling require a balance between osteoclast and osteoblast activity [26]. Osteomodulin (OMD), or osteoadherin, is part of the leucinerich repeat proteins (SLRPs) located in the extracellular matrix. OMD is expressed by osteoblasts and is involved in the regulation of bone formation [27]. OMD has also been shown to regulate the diameter and shape of 
collagen fibrils [28]. The SRM findings presented here for both osteocalcin and osteomodulin in $\mathrm{HO}$ are consistent with the cell data from resected human $\mathrm{HO}$ bone that expressed the osteoblast phenotype (type I collagen) [29]. Other investigators have reported that collagen expression was increased in tissue from wounds with HO for COL10A1, COL4A3, and COL11A1 $[4,30]$.

\section{Conclusions}

This study is the first reported SRM-MS analysis of serum from individuals with and without heterotopic ossification. Differences in the serum proteomic profile between healthy and diseased subjects were identified. Furthermore, our results indicate that normal wound healing signals can impact the ability to identify biomarkers, and a multi-protein panel assay, including osteocalcin preproprotein, osteomodulin precursor, and collagen alpha-1(v) chain isoform 2 preprotein, may provide a solution for $\mathrm{HO}$ detection and monitoring. The proteomic analysis within this report focuses on protein abundance, ignoring protein posttranslational modifications (PTM). Of interest, osteocalcin has several amino acid residues that are susceptible to PTM that influence the function of this protein [31]. Future studies are planned to identify the presence and potential of differentially regulated PTMs in HO.

\section{Additional files}

Additional file 1: Selection reaction monitoring (SRM) peptide transition parameters for protein candidates. Transition parameters and retention times of the 30 peptides were confirmed individually using an Agilent 6495 Triple Quadrapole Mass Spectrometer for both doubly and triply charged precursor ions. Five or 6 transitions per peptide precursor were selected for SRM analysis. In total, 350 transitions were optimized to identify and quantify 30 peptides. SRM target protein names, representative proteotypic peptide sequences, and SRM transition parameters are provided. (XLSX $68 \mathrm{~kb}$ )

Additional file 2: iTRAQ serum gene ontology enrichment analysis. All proteins quantified in the serum via ITRAO were analyzed using BiNGO and cytoscape on June 23, 2016. An over-representation analysis, hypergeometric test with a Benjamini \& Hochberg False Discovery Rate (FDR) correction, was executed with a significance level of $<0.05$. (XLSX $130 \mathrm{~kb})$

Additional file 3: Scatterplots selection reaction monitoring assay. Plot matrix of SRM peptide abundance in blood serum from heterotopic positive (blue) and negative (gold) subjects. (PNG $3461 \mathrm{~kb}$ )

\section{Abbreviations}

ALPL: Alkaline phosphatase; AUC: Area under the curve; BAMC: Brooke Army Medical Center; BGLAP: Osteocalcin preprotein; COL1A2: Collagen alpha-2(I) chain precursor protein; COL5A1: Collagen alpha-1(V) chain; FDA: Food and Drug Administration; GLM: Generalized linear model; HO: Heterotopic ossification; HO -: Heterotopic ossification, disease negative; $\mathrm{HO}+$ : Heterotopic ossification, disease positive; HPLC: High-performance liquid chromatography; IBSP: Bone sialoprotein 2 precursor; IRB: Institutional Review Board; iTRAQ: Isobaric tags for relative and absolute quantitation; KEGG: Kyoto Encyclopedia of Genes and Genomes; LPIN2: Phosphatidate phosphatase; MMP: Matrix metalloproteinase; MS: Mass spectrometry; MSE: Mean square error; ND: Non-disease; NSAID: Nonsteroidal antiinflammatory drug; OEF: Operation Enduring Freedom; OIF: Operation Iraqi Freedom; OMD: Osteomodulin; PPM1J: Protein phosphatase 1J; PTM: Posttranslational modifications; RF: Random forest; ROC: Receiver operating characteristic; RRP12: RRP12-like protein; SCl: Spinal cord injury; SRMMS: Selection reaction monitoring mass spectrometry; SVM: Support vector machine learning; TBI: Traumatic brain injury; TCEP: Tris-(2-carboxyethyl) phosphine; TFA: Trifluoroacetic acid; TRAF3: TRAF3-interacting protein 1; USAMRMC: US Army Medical Research and Materiel Command

\section{Acknowledgements}

The authors thank Ryan V. Wang, PhD, MHS, MS, MyOmicsDx, Inc. for expertise and assistance in the development of the SRM-MS diagnostic panel and Thomas T. Wood, MD, San Antonio Military Medical Center for assistance with determining patient $\mathrm{HO}$ status.

\section{Funding}

The research was funded by the Department of Defense, grant W81-WXH-10-20139, LEE Co-PI. ELC was supported by the Nemours Biomedical Research department and the National Institute of General Medical Sciences of the National Institutes of Health, grant U54-GM104941 (DE-CTR).

\section{Availability of data and materials}

The raw mass spectrometry data, both ITRAQ and SRM, are available via MyOmixDx, Inc., Towson, MD, USA. Restrictions apply to the availability of these data, which were used under license for the current study, and so are not publicly available. Data are available from the authors upon reasonable request and with permission of MyOmixDX, Inc. Processed MS data are included in the manuscript, and raw data files are available from the corresponding author on reasonable request.

\section{Authors' contributions}

LEE contributed to the conception and design of the study, data interpretation, and manuscript preparation. ELC contributed to the bioinformatics analysis, data interpretation, and manuscript and figure preparation. PMO contributed to the analysis of the data and manuscript revision. JTW contributed to the experimental design, data analysis and interpretation, and manuscript preparation. All authors read and approved the final version and agreed to be accountable for all aspects of the work in ensuring that questions related to the accuracy or integrity of any part of the work are appropriately investigated and resolved.

\section{Competing interests}

The authors declare that they have no competing interests.

\section{Consent for publication}

Not applicable.

\section{Ethics approval and consent to participate}

This research was performed in accordance with the Declaration of Helsinki. Subjects were enrolled at Brooke Army Medical Center (BAMC) after giving consent. The study protocol and consent received Institutional Review Board (IRB) from BAMC IRB, Daemen College IRB, and the US Army Medical Research and Materiel Command (USAMRMC) Human Research Protection Office.

\section{Publisher's Note}

Springer Nature remains neutral with regard to jurisdictional claims in published maps and institutional affiliations.

\section{Author details}

${ }^{1}$ Center for Wound Healing Research, Natural Sciences, Daemen College, Amherst, NY 14226, USA. ${ }^{2}$ Bioinformatics, Nemours Biomedical Research, Nemours Alfred I. duPont Hospital for Children, Wilmington, DE 19803, USA. ${ }^{3}$ San Antonio Military Medical Center, San Antonio, TX, USA. ${ }^{4}$ Center for Bioinformatics \& Computational Biology, University of Delaware, Newark, DE 19711, USA.

Received: 7 September 2016 Accepted: 12 April 2017 Published online: 04 May 2017

\section{References}

1. Potter BK, Burns TC, Lacap AP, et al. Heterotopic ossification following traumatic and combat-related amputations. Prevalence, risk factors, and preliminary results of excision. J Bone Joint Surg Am. 2007:89:476-86. doi:10.2106/JBJS.F.00412. 
2. Forsberg JA, Pepek JM, Wagner S, et al. Heterotopic ossification in high-energy wartime extremity injuries: prevalence and risk factors. J Bone Joint Surg Am. 2009;91:1084-91. doi:10.2106/JBJS.H.00792.

3. Alfieri KA, Forsberg JA, Potter BK. Blast injuries and heterotopic ossification. Bone Jt Res. 2012;1:174-9. doi:10.1302/2046-3758.18.2000102.

4. Davis TA, O'Brien FP, Anam K, et al. Heterotopic ossification in complex orthopaedic combat wounds: quantification and characterization of osteogenic precursor cell activity in traumatized muscle. J Bone Joint Surg Am. 2011;93:1122-31. doi:10.2106/JBJS.J.01417.

5. Potter BK, Forsberg JA, Davis TA, et al. Heterotopic ossification following combat-related trauma. J Bone Joint Surg Am. 2010;92 Suppl 2:74-89. doi:10.2106/JBJS.J.00776.

6. Foruria AM, Lawrence TM, Augustin S, et al. Heterotopic ossification after surgery for distal humeral fractures. Bone Joint J. 2014;96-B:1681-7. doi:10.1302/0301-620X.96B12.34091.

7. Ranganathan K, Loder S, Agarwal S, et al. Heterotopic ossification: basicscience principles and clinical correlates. J Bone Joint Surg Am. 2015;97: 1101-11. doi:10.2106/JBJS.N.01056.

8. Babuin L, Jaffe AS. Cardiac injury. Review. 2005;173:1191-202.

9. Toffoli AM, Gautschi OP, Frey SP, et al. From brain to bone: evidence for the release of osteogenic humoral factors after traumatic brain injury. Brain Inj. 2008;22:511-8. doi:10.1080/02699050802158235.

10. Cadosch D, Toffoli AM, Gautschi OP, et al. Serum after traumatic brain injury increases proliferation and supports expression of osteoblast markers in muscle cells. J Bone Joint Surg Am. 2010;92:645-53. doi:10.2106/JBJS.I.00097.

11. Lee J-H, Cho J-Y. Proteomics approaches for the studies of bone metabolism. BMB Rep. 2014;47:141-8. doi:10.5483/BMBRep.2014.47.3.270

12. Anderson L. Quantitative mass spectrometric multiple reaction monitoring assays for major plasma proteins. Mol Cell Proteomics. 2005;5:573-88. doi:10.1074/mcp.M500331-MCP200.

13. Lange $V$, Malmstrom JA, Didion J, et al. Targeted quantitative analysis of Streptococcus pyogenes virulence factors by multiple reaction monitoring. Mol Cell Proteomics. 2008;7:1489-500. doi:10.1074/mcp.M800032-MCP200.

14. Li X, Hayward C, Fong P-Y, et al. A blood-based proteomic classifier for the molecular characterization of pulmonary nodules. Sci Transl Med. 2013;5: 207ra142. doi:10.1126/scitrans/med.3007013.

15. Wisniewski JR, Zougman A, Nagaraj N, Mann M. Universal sample preparation method for proteome analysis. Nat Methods. 2009;6(5):359-62. doi:10.1038/nmeth.1322.

16. Smoot ME, Ono K, Ruscheinski J, et al. Cytoscape 2.8: new features for data integration and network visualization. Bioinformatics. 2011;27:431-2. doi:10.1093/bioinformatics/btq675.

17. Wu G, Feng $X$, Stein L. A human functional protein interaction network and its application to cancer data analysis. Genome Biol. 2010;11:R53. doi:10.1186/gb-2010-11-5-r53.

18. Maere S, Heymans K, Kuiper M. BiNGO: a Cytoscape plugin to assess overrepresentation of gene ontology categories in biological networks. Bioinformatics. 2005;21:3448-9. doi:10.1093/bioinformatics/bti551.

19. Supek F, Bošnjak M, Škunca N, Šmuc T. REVIGO summarizes and visualizes long lists of gene ontology terms. PLoS One. 2011;6:e21800. doi:10.1371/ journal.pone.0021800.

20. MacLean B, Tomazela DM, Shulman N, et al. Skyline: an open source document editor for creating and analyzing targeted proteomics experiments. Bioinformatics. 2010;26:966-8. doi:10.1093/bioinformatics/btq054.

21. Edsberg LE, Wyffels JT, Brogan MS, Fries KM. Analysis of the proteomic profile of chronic pressure ulcers. Wound Repair Regen. 2012;20:378-401. doi:10.1111/ j.1524-475X.2012.00791.X.

22. Nauth A, Giles E, Potter BK, et al. Heterotopic ossification in orthopaedic trauma. J Orthop Trauma. 2012;26:684-8. doi:10.1097/BOT.0b013e3182724624.

23. Wilkinson JM, Stockley I, Hamer AJ, et al. Biochemical markers of bone turnover and development of heterotopic ossification after total hip arthroplasty. J Orthop Res. 2003;21:529-34. doi:10.1016/S0736-0266(02)00236-X.

24. Patti A, Gennari L, Merlotti D, et al. Endocrine actions of osteocalcin. Int J Endocrinol. 2013;2013:1-10. doi:10.1155/2013/846480.

25. Chauveau C, Devedjian J-C, Blary M-C, et al. Gene expression in human osteoblastic cells from normal and heterotopic ossification. Exp Mol Pathol. 2004;76:37-43.

26. Ninomiya K, Miyamoto T, Imai J, et al. Osteoclastic activity induces osteomodulin expression in osteoblasts. Biochem Biophys Res Commun. 2007;362:460-6. doi:10.1016/j.bbrc.2007.07.193.
27. Sommarin $Y$, Wendel $M$, Shen $Z$, et al. Osteoadherin, a cell-binding keratan sulfate proteoglycan in bone, belongs to the family of leucine-rich repeat proteins of the extracellular matrix. J Biol Chem. 1998;273:16723-9.

28. Tashima T, Nagatoishi S, Sagara H, et al. Osteomodulin regulates diameter and alters shape of collagen fibrils. Biochem Biophys Res Commun. 2015; 463:292-6. doi:10.1016/j.bbrc.2015.05.053.

29. Handschin AE, Egermann M, Wedler $V$, et al. A comparative analysis of phenotype expression in human osteoblasts from heterotopic ossification and normal bone. Langenbeck's Arch Surg. 2006;391:376-82. doi:10.1007/ s00423-005-0021-5.

30. Evans KN, Potter BK, Brown TS, et al. Osteogenic gene expression correlates with development of heterotopic ossification in war wounds. Clin Orthop Relat Res. 2014;472:396-404. doi:10.1007/s11999-013-3325-8.

31. Gundberg CM, Lian JB, Booth SL. Vitamin K-dependent carboxylation of osteocalcin: friend or foe? Adv Nutr An Int Rev J. 2012:3:149-57. doi:10.3945/an.112.001834.

\section{Submit your next manuscript to BioMed Central and we will help you at every step:}

- We accept pre-submission inquiries

- Our selector tool helps you to find the most relevant journal

- We provide round the clock customer support

- Convenient online submission

- Thorough peer review

- Inclusion in PubMed and all major indexing services

- Maximum visibility for your research

Submit your manuscript at www.biomedcentral.com/submit
Biomed Central 